\title{
New Prospects in High Energy Astrophysics
}

\author{
KIPAC \\ Stanford \\ CA 94025 \\ USA
}

Roger Blandford

\begin{abstract}
Recent discoveries using TeV, X-ray and radio telescopes as well as Ultra High Energy Cosmic Ray arrays are leading to new insights into longstanding puzzles in high energy astrophysics. Many of these insights come from combining observations throughout the electromagnetic and other spectra as well as evidence assembled from different types of source to propose general principles. Issues discussed in this general overview include methods of accelerating relativistic particles, and amplifying magnetic field, the dynamics of relativistic outflows and the nature of the prime movers that power them. Observational approaches to distinguishing hadronic, leptonic and electromagnetic outflows and emission mechanisms are discussed along with probes of the velocity field and the confinement mechanisms. Observations with GLAST promise to be very prescriptive for addressing these problems.
\end{abstract}

\section{PARTICLE ASTROPHYSICS IN THE $21^{S T}$ CENTURY}

It is worth reminding ourselves at the start what an enormous fraction of the electromagnetic spectrum is commanded by "High Energy Astrophysics" . Conventionally this involves photons from $\sim 0.1 \mathrm{keV}$ to the highest energy yet detected $\sim 100 \mathrm{TeV}$, a span of 33 octaves of energy. We can add a good share of the ten octave radio spectrum as well as the cosmic ray, neutrino, gravitational radiation spectra then this is more than half the available territory. Of course, there is far more going on physically in the single octave used by optical astronomers, but the high energy region contains important fundamental energies too. First, there are all the lines associated with heavy elements in the X-ray spectrum which allow us to make detailed inferences about the physical conditions - density, temperature and so on as well as the bulk velocity of cosmic sources. At somewhat higher energy are the low energy nuclear emission lines, e.g. of cobalt and titanium. Next there is the electron-posittron rest mass $\sim 0.5 \mathrm{MeV}$ which is the rest energy of a simple pair annihilation $\gamma$-ray. This is followed by the intermediate nuclear energy lines of deuterium and aluminium in the $\mathrm{MeV}$ range. Next comes the pion mass which gives a scale to the broad hadronic $\gamma$ - ray spectrum around $\sim 100 \mathrm{MeV}$. Finally there is the proton mass which marks a physics scale in many particle acceleration processes This may be made manifest in observed electromagnetic spectra. Although high

\footnotetext{
${ }^{1}$ It is not possible in a review this broad to begin to do full justice to the immense bibliography in this area which represents a considerable amount of hard work and thought over more than forty years. Therefor I have just cited a few very general references at the end of this article where good entries into the literature may be found.
} 
energy astrophysics involves observing throughout the electromagnetic spectrum from $\sim 100 \mathrm{neV}$ radio waves to $\sim 100 \mathrm{TeV} \gamma$-rays, it also embraces, or one day will embrace, the non-electromagnetic cosmic neutrino and gravitational radiation spectra.

\section{TeV Astrophysics}

These are exciting times in $\gamma$-ray astrophysics. TeV scale (typically above $100 \mathrm{GeV}$ ) astronomy has come of age. [1] H.E.S.S., MAGIC and VERITAS -three ground-based air Cerenkov facilities, located in Namibia, the Canary Islands and the United States respectively, have discovered almost a hundred sources and several new source classes. This is well beyond the handful of sources discovered by the Whipple Observatory. The technique can only be used during dark time when a $\sim 10 \mathrm{~ns}$ flash created by Cerenkov emission from a $\gamma$-ray induced shower is observed stereoscopically from two or more telescopes on the ground. The primary gamma ray energy and direction can be determined to about ten percent and $\sim 5$ arcmin respectively. An alternative approach is the water Cerenkov method, through which secondary electrons are detected in one or several water tanks. Water Cerenkov detectors work best at high energy, and have a much larger field of view and duty cycle than air Cerenkov detectors but smaller collecting area.

\section{GeV Astrophysics}

Even greater advances are expected in $\mathrm{GeV}$ astrophysics with the successful launch of AGILE and the expected launch of GLAST ${ }^{2}$ where the principle LAT instrument can detect photons from $\sim 0.02-300 \mathrm{GeV}$. [1] GLAST works by tracking pairs and their progeny created by a primary gamma ray incident upon a Silicon strip tracker which fixes the direction. A calorimeter measures the energy. Up to ten thousand cosmic rays have to be rejected for every $\gamma$-ray detected.

\section{Gamma ray Sources}

Just as happened with radio astronomy, the $\gamma$-ray sky is turning out to be much more interesting than predicted. Firstly, the most numerous sources are blazars - relativistic jets created by spinning, massive black holes in Active Galactic Nuclei (AGN) that happen to be directed towards us. Up to ten thousand such sources should be detected over a ten year GLAST lifetime. We already know that their $\gamma$-ray emission is highly variable - two minute variation has already been reported. We hope to understand the "cosmic engineering" of jets and settle longstanding controversy about their composition, speed

\footnotetext{
${ }^{2}$ Launched on June 112008
} 
and confinement. Other types of galaxy, for example, Ultra Luminous InfraRed Galaxies (ULIRGs), are prime candidates for detection.

Gamma Ray Bursts (GRBs) have already been observed by EGRET but far more should be detected by GLAST, up to a hundred per year [3]. They come in three basic types. The long duration ( $>2 \mathrm{~s}$ ) bursts are associated with core collapse supernovae and can be seen to great cosmological distance [6]. Short duration bursts are suspected to be due to binary neutron star coalescence and the "Soft Gamma Repeaters" (SGRs) are nearby neutron stars endowed with $10^{14-15} \mathrm{G}$ magnetic fields that undergo violent flares. Much remains to be understood about GRBs and their afterglows.

Radio pulsars, which are neutron stars associated with lower, (typically $10^{12} \mathrm{G}$ fields that are mostly easily detected through their coherent radio emission. They pulse much more powerfully at $\gamma$-ray energies and up to a hundred could be detected. Their electrical engineering is quite controversial and the opportunity now is to understand how they shine.

Many SuperNova Remnants (SNR) are being detected as TeV sources verifying that non-relativistic shock fronts are able to accelerate protons and electrons to prodigious energy, perhaps as high as $1 \mathrm{PeV}$ [5]. It has also been appreciated more recently that magnetic field is amplified too. It is hoped that $\mathrm{GeV}$ observations will help elucidate the physical details. In a subset of remnants, called Pulsar Wind Nebulae (PWN), emission is seen also from a central neutron star and its wind.

Other anticipated sources are microquasars, (black holes and neutron stars that create miniature relativistic jets), winds from young hot stars and the hot gas in clusters of galaxies. There will also be an extensive, diffuse emission associated with cosmic rays propagating through the interstellar medium. These will create a background against which the most important signal that could be seen - a $\gamma$-ray spectral feature associated with dark matter annihilation - will have to be investigated.

However, as was the case with EGRET, there are likely to be several populations of unidentified sources that may be associated with new classes of astronomical emitter.

\section{Multi-wavelength Observing}

$\gamma$-ray astrophysics has evolvved from an emphasis on basic detection to programs that explore foundational questions in both physics and astronomy. Both endeavors require that $\gamma$-ray observations by augmented by astronomical observations throughout the electromagnetic spectrum. VLBI observations (including those by Astro-G due for launch in 2012) will be especially important for understanding the blazars as they will probe radii only hundreds of times larger than those associated with the $\gamma$-rays. Single dish radio telescopes will monitor the pulsars and provide the reference for locating the $\gamma$-ray emission site. One of the cosmological fruits of gamma-ray astronomy will be to measure the integrated background emission of galaxies as a function of cosmic time. This will be closely compared with direct infrared measurements from Spitzer andJWST. Optical spectroscopy with ten meter class telescopes will be needed to study many of the source identifications. X-ray and $\mathrm{TeV}$ observations of supernova remnants have already transformed our understanding of cosmic particle acceleration. We await 
the GLAST observations to fill in the gap. In addition, Swift X-ray observations will be used to identify GRBs. In particular, it will overlap with the LAT a quarter of the time. These are just some of the ways that multi-wavelength observations are being used and will be used to bring out the full value of high energy observatories.

\section{UHE Cosmic Rays}

The observed cosmic ray spectrum extends from $\sim \mathrm{MeV}$ energies through $\sim \mathrm{GeV}$ energies where the effects of solar modulation have to be removed and then extends with spectrum $N(E) \propto E^{-2.6}$ up to the "knee" at $\sim \mathrm{PeV}$ energy where it steepens before flattening at the ankle at $\sim \mathrm{EeV}$ energy to the highest energies recorded approaching $\sim \mathrm{ZeV}$ energy. The highest energy particles have $\sim 50 \mathrm{~J}$ energy and lag behind a photon by a speed of order a km per Hubble time! The UHE cosmic rays above $\sim 1 \mathrm{EeV}$, present a challenge to astrophysics. They have to be accelerated and propagate in the presence of microwave background photons which look like $\sim 100 \mathrm{MeV} \gamma$-rays in the cosmic ray rest frame and which can create pions. The prediction is that the cosmic ray spectrum should exhibit a "GZK" break around $\sim 100 \mathrm{EeV}$. This feature has now been reported by the Auger collaboration. Another, far more tentative, claim has been the statistical association of cosmic ray arrival directions with active galaxies. If substantiated by more data, we will be doing cosmic ray astronomy!

\section{PARTICLE ACCELERATION}

\section{Hadrons vs leptons}

A fundamental question that has to be answered in many $\gamma$-ray sources is whether the emission is hadronic or leptonic, via pion decay or inverse Compton emission, respectively. In the case of SNRs, there is clear evidence for nonthermal electron synchrotron emission by $\sim 10-100 \mathrm{TeV}$ electrons [5]. This emission has recently been shown to be variable on year timescales which is argued to be due to radiative cooling implying that the magnetic field is amplified strongly while the particles are accelerated, presumably at the shock front. This, in turn, depresses the relativistic electron density requiring the $\gamma$-ray emission which has been detected up to $\sim 100 \mathrm{TeV}$ energies, to be hadronic. A spatial correlation between the X-rays and $\gamma$-rays is also observed which is most easily interpreted if the relativistic electrons and protons, the gas density and the magnetic field are all correlated. Future GeV observations should be capable of substantiating or refuting this chain of argument for hadronic emission in SNR by detecting spectral evidence of pion emission.

Turning to the blazars, the observation of X-ray synchrotron emission by $\sim 100 \mathrm{TeV}$ relativistic electrons with very short cooling times implies that the particle acceleration must be continuous along the jet instead of being localised in separated shock fronts. This is supported by reports of rapidly variable $\mathrm{TeV}$ emission from powerful blazars which is most easily explained as inverse Compton scattering of external photons close 
to the massive black hole which powers the relativistic jet. The balance of the evidence is that blazar $\gamma$-ray emission is leptonic.

\section{Accelerators}

\section{Diffusive Shock Acceleration}

Cosmic accelertors either act stochastically where particles gain and lose energy in small increments with the gains dominating the losses -an idea associated with Fermi - or systematically where a large potential difference is maintained in the presence of a plasma. Diffusive shock acceleration is an example of stochastic acceleration. In its simplest manifestation, protons and electrons are scattered across a non-relativistic shock with compression ratio $r$ so that the energy gained in bouncing off an approaching scattering center ahead of the shock exceeds that lost behind the shock front, In the simplest, test particle, approximation, the transmitted relativistic momentum particle distribution function is given by

$$
f_{+}(p)=q p^{-q} \int_{0}^{p} d p^{\prime} p^{q-1} f_{-}\left(p^{\prime}\right)
$$

where $f_{-}(p)$ is the incident distribution function far upstream of the shock and $q=$ $3 r /(r-1)$. So, for a strong adiabatic shock with $r=4, q=4$. The observed slope in the GeV-PeV range is $q \sim 4.6$ and if we take into account the energy dependence of the escape time, $q \sim 4.2$ at the source. This is remarkably good agreement. Furthermore, the Green's function is linear and it is reasonable to suppose that the rate of particle injection will increase until the pressure associated with the transmitted distributions reacts dynamically on the underlying non-relativistic gas. In other words the acceleration mechanism is very efficient.

This agreement is probably too good to be true and several important questions are raised. The first is the nature of the magnetic inhomogeneities. Originally these were thought to be Alfvén waves that were self-excited by streaming cosmic rays as a result of a linear instability. The cosmic rays only diffuse parallel to the magnetic field and However it is easy to see that if the mean magnetic field strength has a typical interstellar value of a few microgauss, then the magnetic fluctuations must be nonlinear. It is common to suppose that the diffusion follows the Bohm model with coefficient $D \sim r_{L} v \propto p / B$, where $r_{L}$ is the Larmor radius. The distance that a particle streams ahead of the shock front is $x(p) \sim D / u$. where $u$ is the shock speed. This increases roughly linearly with energy. The next complication is that if $q \sim 4$, the highest energy particles will carry a significant fraction of the pressure and can decelerate the incoming gas, weakening the shock front and steepening the spectrum. This seems like a stable way for the cosmic rays to control their own injection but the details turn out to be hard to understand. The next challenge is to understand what happens when $v \sim u$. This is relevant to particle injection and to the structure of relativistic shocks. The final issue is the maximum particle energy accelerated by a shock. With the above scalings, this is $\sim e u B R$, where $R$ is the radius of the shock front. Observing $100 \mathrm{TeV} \gamma$-rays implies 
that we must accelerate $\sim 0.3 \mathrm{PeV}$ cosmic rays. This, in turn, implies that if particles are accelerated at shock fronts, then the magnetic field strength in the acceleration region must exceed $\sim 200 \mu \mathrm{G}$. (This conclusion is, within reason, independent of the acceleration mechanism.)

How can magnetic field amplification be incorporate into diffusive shock acceleration? Several proposals have been made already but it seems most likely that it is the cosmic rays themselves that are responsible. One particular way that this can happen is through a "magnetic bootstrap" mechanism ][1]. In outline, this relies upon the fact that the highest energy particles to be accelerated stream furthest ahead of the shock front. When they stream a distance of order the shock radius ahead of the front, they become unlikely to return for further acceleration and this condition determines the upper energy cut off. These particles are the first manifestation of the approaching shock for the interstellar medium and because they are partly escaping they have a quite unstable distribution function and their pressure will dominate the ambient interstellar thermal and magnetic pressure. Firehose modes will grow fast enough to allow the magnetic field to become nonlinear in strength and provide the background field that will scatter lower energy particles with smaller Larmor radii closer to the shock front. (Note that the distinction between quasi-parallel and quasi-perpendicular shocks has now disappeared.) There are several distinctive observable implications of this model.

Although supernova remnants provide our best laboratory for studying processes like this, shock structure should be quite generic. For example the shocks that first increased the entropy of primordial gas in the early universe and which ultimately fell into deep gravitational potential wells should also have accelerated $\mathrm{GeV}$ cosmic rays and generated large magnetic fields. Clusters of galaxies ought to be gamma ray and electron synchrotron sources.

\section{Systematic Acceleration}

The most commonly invoked form of systematic acceleration is some form of unipolar induction like a Faraday disk. This can be associated with neutron stars, black holes or accretion disks. On general ground the EMF induced is $\mathscr{E} \sim \Omega \Phi$ where $\Omega$ is the characteristic angular frequency and $\Phi$ is the magnetic flux. Under relativistic conditions, which are optimal for particle acceleration, the current flowing is $I \sim \mathscr{E} / Z_{0}$, where $Z_{0}$ is the impedance of free space $377 \Omega$. The maximum power is generically $L \sim \mathscr{E} I \sim 10^{47}(\mathscr{E} / 1 \mathrm{ZV})^{2} \mathrm{erg} \mathrm{s}^{-1}$. Acceleration of UHE cosmic rays requires sources as luminous as $\sim 10^{46} \mathrm{erg} \mathrm{s}^{-1}$ of which the most plausibnle candidate is an AGN. The way that this happens brings out some general principles that are also applicable to GRBs. Suppose that a massive black hole and its attendant accretion disk acts as a unipolar inductor and a current $\sim 1$ EA flows. The current will ultimately close at some distance from the source. If it does this by passing through a resistive load $\sim 100 \Omega$, then the power will go into heat leading to a thermally driven jet. Alternatively, the current can cross toroidal magnetic field lines and create a Lorentz force which accelerates a wind non-dissipatively. Either outcome represents the conversion of electromagnetic Poynting flux into mechanical momentum. If this happens very close to the black hole, then 
the observed emission will be from a fluid dynamical jet; if not, the jet is described as electromagnetic.

\title{
RELATIVISTIC OUTFLOWS
}

\begin{abstract}
AGN
The oldest known and most numerous jets are associated with massive black holes (of mass $M$ ) residing in the nuclei of normal galaxies [4]. A subset of these black holes, quite possibly those that are spinning rapidly and accreting mass relatively slowly in comparison with the Eddington rate, $4 \pi G M m_{p} / \sigma_{T} c$, produce prodigiously powerful, collimated relativistic outflows called jets. The Lorentz factors associated with these outflows is typically $\Gamma \sim 10$ as measured by the phenomenon of superluminal expansion. Unfortunately, going beyond this with confidence has proven difficult. The hope, and I think it is a serious one, is that imminent observations coupled with more sophisticated numerical simulations will lead to answers.
\end{abstract}

\section{Emission Location}

Perhaps the most straightforward question to pose and the easiest to imagine answering conclusively is the location of the sources at different wavelengths. The radio emission is widely supposed to be synchrotron radiation by $\sim \mathrm{GeV}$ electrons. The observed spectra of jet cores are quite flat $S_{V} \propto v^{0}$, roughly. This is generally supposed to arise from an inhomogeneous source. There is a radio photosphere behind which the jet is opaque to synchrotron self-absorption. As the radio frequency is increased, we see down to smaller jet radii. Under these circumstances, the brightness temperature of the photospheric radiation $T^{\prime}$ measured in the comoving frame at frequency $v^{\prime}$ of the outflowing jet is given approximately by $T^{\prime} \sim \gamma^{\prime} m_{e} c^{2} / 3 k$, where $\gamma^{\prime} m_{e} c^{2}$ is the energy measured in the comoving frame of the electrons emitting at frequency $v^{\prime}$. The frequency and brightness temperature measured in the frame of the galaxy will be boosted so that $v \sim \Gamma v^{\prime}$, $T \sim \Gamma T^{\prime}$. We can also evaluate the magnetic field in the comoving frame, $B^{\prime}$. Note that although the emission from the core is Doppler-boosted, the flow is stationary. There is basically one degree of freedom in making a model like this and that is sometimes removed by measuring the inverse Compton emission by the same electrons scattering synchrotron photons. This is one area where the upcoming suite of multi-wavelength observations motivated by GLAST should make a big difference.

The radio variability that is measured by VLBI observations is thought to be associated with optically thin emission sites moving relative to the core with a speed related to the jet velocity. They fade with time and are replaced by fresh features moving out from the core. The gamma ray emission is also variable and is probably inverse Compton emission. Both $\mathrm{TeV}$ and $\mathrm{GeV}$ emission is commonly seen and both can be quite variable. The simplest expectation is that the $\gamma$-ray emission is produced at smaller radii than the shortest radio wavelength radio photosphere and the $\mathrm{TeV}$ emission which requires higher energy particles originates within the $\mathrm{GeV}$ emission. A second possibility 
is that all the inverse Compton emission originate from the same site, presumably moving outward. it is the local nature of the particle acceleration that is responsible.The third possibility - that the $\mathrm{TeV}$ emission originate outside the $\mathrm{GeV}$ emission - is also possible. This case arises if there is also a $\gamma$-ray photosphere due to the pair production opacity caused by the high density of $\mathrm{keV}$ (at $\mathrm{GeV}$ energy) or $\mathrm{eV}$ (at $\mathrm{TeV}$ energy) photons. The photospheric radius will, assuredly, increase with energy according to this scheme. These three cases can be distinguished observationally by monitoring the variability and seeing if the higher energy photons precede or follow the lower energy photons.This question may best be answered statistically.

\section{Jet Velocity}

The next question to address is the nature of the velocity field in the jet. This is crucially important because Doppler beaming is such a powerful amplifier. If we consider a single source moving with constant velocity $\vec{\beta}$ and spectral index $\alpha=$ $-d \ln S_{V} / d \ln v$ and an observer direction $\vec{n}$, then the observed flux $S_{V} \propto \mathscr{D}^{3+\alpha}$ where $\mathscr{D}=[\Gamma(1-\vec{\beta} \cdot \vec{n})]^{-1}$ is the Doppler factor. (For a stationary flow the exponent is $2+\alpha$.) An approaching source with $\alpha=0.5$ is $(2 \Gamma)^{7} \sim 10^{9}$ for $\gamma \sim 10$ times brighter than a receding source. Meanwhile the dynamic range - the ratio of largest and smallest intensity measurable - of a typical VLBI observations is $\sim 10-100$. As this graphic example makes clear, what we actually observe is dominated by possibly insignificant parts of the source that are moving in our direction. Conversely, models of radio sources that assume monolithic sources lead to wildly different beaming distributions from those that have more realistic fluid characteristics like boundary layers, entrainment and turbulent backflows. It is not clear when jets accelerate as adiabatic flows expanding down a density gradient do, and when they decelerate as they will if they interact with their surroundings. It is, however, observed that the low power "FR1" sources that turn into subsonic plumes start off with relativistic speeds.

\section{Emission Mechanisms}

Somewhat surprisingly, the emission mechanism is still contentious and alternatives to the traditional pattern of synchrotron emission at low energy and inverse Compton scattering at high energy are still seriously discussed. The most common alternative to synchrotron emission is some form of coherent cyclotron maser. The motivation for doing this is that the brightness temperature inferred on the basis of radio monitoring implies that the radiative efficiency is unreasonably low, through the chain of argument outlined above. Masers are not a panacea, though, because high brightness radiation may be subject to nonlinear induced Compton and stimulated Raman scattering which may inhibit its emergence from the source. X-ray emission is mixed. In some jets like M87, there are compelling arguments that we are seeing synchrotron emission by $\sim 100 \mathrm{TeV}$ electrons, while in other sources, the X-rays are created by inverse Compton scattering of microwave background or synchrotron photons. In the $\gamma$-ray region, the alternative of 
a hadronic jet, where all the energy is carried by the $\sim 100 \mathrm{TeV}$ protons, and lost through collisions with stationary gas or ambient radiation. The "smoking gun" of a hadronic jet is the VHE neutrino emission that could be seen, in principle, by a facility like IceCube. Even in the context of inverse Compton scattering by electrons, there is a choice to be made. Either the photons that are scattered are created internally - the Synchrotron SelfCompton (SSC) model - or they are Thomson scattered from the accretion disk into the jet - the External Compton (EC) model. The former choice is favored for low power jets and the latter for high power jets, though all of this needs corroboration.

\section{Particle Acceleration}

There are also important questions about the particle acceleration. It has been tempting to invoke diffusive shock acceleration, as practiced in supernova remnants, for jets. However, there are at least three reasons why alternative mechanisms may be at work. The first is that most of the shocks are likely to be relativistic and although efficient, the details are likely to be qualitatively different from what happens at supernova remnants. The second is that if the shocks are nonrelativistic, then they accelerate protons thirty time more efficiently than electrons and it is the electrons that radiate. The third problem is the most prescriptive. The cooling lengths of the high energy electrons responsible for $\mathrm{X}$-ray synchrotron radiation are very short - light decades- and far smaller than the jet widths and so the emission would have to be confined to the shock fronts. However, the jet emission appears to have a large, diffuse component and cannot be associated with widely-spaced shock fronts. Fortunately, there is a variety of alternative second-order, stochastic emission mechanisms plus reconnection which, although inefficient in a nonrelativistic context are likely to be quite effective and able to act homogeneously in a relativistic flow. These processes need further study.

\section{Composition}

The next question to address is that of jet composition. Three answers have been given, leptonic, hadronic and electromagnetic. Taking these in turn, leptons, (pair plasma) cannot carry the bulk of the jet momentum close to the black hole. The radiative drag is far too large. It can however dominate jets further out where the external radiation field is smaller. Hadronic jets can carry the momentum all the way from the black hole. However, the presence of strong polarization at radio frequencies places a lower bound on the density of nonrelativistic and, indeed, relativistic electron-ion plasma in order to avoid Faraday depolarization. (This is not an issue with a pair plasma where the eigenmodes are linear.) There are ways of circumventing this constraint but it does provide a modeling challenge. A second issue concerns bulk Comptonization. This is due to cold electrons accompanying ions moving with bulk relativistic speed. The observed $\mathrm{X}$-ray spectrum furnishes an upper limit on this process which has been used to argue against hadronic jets in some cases. Electromagnetic jets, in which Poynting flux os very important, come in two forms, AC and DC. The former comprises a tangled field that 
has an essentially isotropic pressure tensor when average over large enough volumes. They are similar to fluid jets in their dynamical properties. DC jets carry $\sim$ EA $\left(10^{18} \mathrm{~A}\right)$ currents. They are naturally pinched but have cores where the particle flux dominates and typical models of relativistic "electromagnetic" jets carry at least 90 percent of their power in the form of particles.

Of course, it is not required that there be one answer for all jets or even a given jet at all radii. Indeed, the most natural rationalization of the observations of jets on all scales is that they are formed essentially electromagnetically near the black hole with the pairs primarily supplying the current, The pair density is initially low because annihilation is more efficient than pair production and particle acceleration. Eventually the balance shifts as the jet expands and the pairs become dynamically dominant. Meanwhile, ambient plasma and outflowing gas from the accretion disk are entrained into the jet from the outside and the jet becomes ion-dominated and moves more slowly.

\section{Confinement}

The final major question to be answered for models of AGN jets is what confines them. The problem is real because the minimum pressure in jets exceeds the maximum external thermal pressure given by X-ray observations in some jets. As alluded to above, a very attractive answer is that they are confined by the hoop stress associated with toroidal magnetic field associated with a jet-parallel electrical current. In this way a small external pressure can confine a much larger jet pressure. Pinches like this are notoriously unstable but exceptions may be made under these circumstances on account of the large kinetic energy of the core and the jet expansion due to the surrounding pressure decreasing with radius. The way to test this model is to seek coherent rotation measure gradients across the jet that would suggest the presence of a large scale magnetic field. There are some hints of this effect, but more of these technically challenging observations are needed. Alternatively jets may be confined very close to the black hole and then propagate ballistically, expanding transversely only at a multiple of the internal sound speed in the co-moving frame. This is not a viable explanation for those jets where the opening angle decreases with increasing radius.

\section{GRBs}

GRBs are stellar explosions at cosmological distances where a significant fraction (at least $\sim 10^{-3}$ ) of a stellar rest mass is released in a matter of seconds. The long bursts, lasting more than a few seconds, are associated with core collapses supernovae, though there are only a few good examples where the supernova explosion has been seen. Short bursts are most commonly supposed to be due to coalescing neutron star binaries, though here the evidence is still poor. Both types of GRB seem to create relativistic beamed jets which reduces the estimate of the total explosion energy. The Lorentz factors of the outflows must typically exceed several hundred in order that the observed $\gamma$-rays avoid pair production on lower energy photons as they escape from the source. 
GRBs are followed by "afterglows" from blast waves expanding into the interstellar medium, relativistic and elongated at first and then decelerating to nonrelativistic quasispherical flows like regular supernova remnants. These are observed from radio through $\mathrm{X}$-ray energies although the particle acceleration and field amplification mechanisms are not well understood. Observations using the Swift satellite have shown that dynamics is quite complex and there is considerable variation in the observations from burst to burst. This should not be surprising as they are ultrarelivistic explosions oberved from all directions.

There are similar unanswered questions about GRB explosions as there are about AGN jets - the composition, the velocity field and the collimation. Most early models invoked a hadronic outflow. The $\gamma$-ray production was commonly attributed to electrons and positrons accelerated at internal shocks. caused by variations in the source. Alternatively electromagnetic models are quite viable and local instabilities can be responsible for the emissivity. EGRET detected a few examples of $\mathrm{GeV}$ emission but $\mathrm{TeV}$ emission has not yet been seen. Observations with GLAST (working in tandem with Swift) should be quite prescriptive. In particular, if prompt $\mathrm{GeV} \gamma$-rays are detected commonly, then this will severely constrain the hadronic jet - internal shock interpretation. Again the nature of the prime mover remains uncertain although it is tempting to associate the burst with black hole formation, either following core collapse or neutron star coalescence. Possible future detectlons of gravitational radiation or neutrinos would also be highly informative.

"Soft Gamma Repeaters" (SGR) constitute a quite different type of GRB. These are assocIated with highly magnetized $\left(\sim 10^{14-15} \mathrm{G}\right)$ neutron stars in our Galaxy or close neighbors like the Magellanic Clouds. The neutron stars are observed to spin comparatively slowly, with rotation periods $\sim 5 \mathrm{~s}$, Giant magnetospheric rearrangements can release up to $\sim 10^{47}$ erg of magnetic energy which creates a relativistic explosion which sweeps up the interstellar medium into a relativistic blast wave. The rise time of the $\gamma$-ray pulse in the best studied example of the Dec 242004 burst in SGR 1806-20 was measured to be only $300 \mu \mathrm{s}$. A rapidly spinning $(\sim \mathrm{kHz}$ frequency magnetar could release GRB level energies in seconds and, as such, provides a plausible alternative prime mover for a long GRB.

\section{Pulsar Wind Nebulae}

Normal young neutron stars, with $\sim 10^{12} \mathrm{G}$ fields and $\sim 10 \mathrm{~Hz}$ spin frequencies are commonly found near the centers of young supernova remnants. The power from pulsar, derived ultimately from the rotational kinetic energy of the star, energises a small "Pulsar Wind Nebula" (PWN) [2]. The most famous example is the Crab Nebula, where it has proven extremely hard to detect the surrounding supernova remnant. PWN are observed from radio to $\gamma$-ray bands up to $\sim 100 \mathrm{TeV}$. They often exhibit jet-like features which may result from collimation of a wind that leaves the pulsar quasi-spherically. These have mostly been modeled as hadronic or leptonic outflows. However, it is now also believed that they could be magnetically collimated. The jet features exhibit instability which is quite reasonable as they are mostly subsonic features being contained within a 
supernova remnant.

\section{PRIME MOVERS}

Although understanding the details of the emission regions is probably a more attainable short-term goal, elucidating the properties of the prime mover is probably the more interesting task. As we have so little confidence in our descriptions of the underlying physical processes, the best way to proceed is inductively, not deductively. New high energy astrophysical facilities, as discussed above, open up many opportunities. The first of these concerns pulsars. As with jets, the first task is to understand the topography of the magnetosphere. There are several candidate sites in the inner and the outer magnetosphere. Fortunately, it is now possible to solve the force-free equations for a dipolar magnetosphere that js probably a good approximation to the more complex multipolar magnetosphere, well away from the surface. GLAST should time up to a hundred pulsars and, by comparing the phase-resolved pulse profiles at radio and gamma ray energies it should be possible to measure the inclination of the spin axis and locate the various emission sites. More TeV and GeV observations of PWN should settle the matter of the degree of magnetization of the outflow and help us assemble a fuller understanding of pulsars in particular and electromagnetic outflows in general. (We are completely confident that the power is extracted from the neutron star by electromagnetic torque as a Poynting flux. The question, again, is where and how do the associated electrical currents close; within the light cylinder, at a termination shock or at the outer boundary of the nebula. The next task is to see how the $\gamma$-ray power relates to the EMF induced by the neutron star and use the pulse profile to piece together the emission mechanism.

Advances in pulsar electrodynamics may then help in developing an understanding of how strongly magnetized relativistic plasma behaves in practice. Issues like whether the currents flow in filaments, sheets or volumetrically, how does magnetic field reconnect and whether or not electromagnetic field can evolve so that the invariant $B^{2}-E^{2}$ changes sign anywhere, leading to rapid dissipation, will all need to be understood. This general understanding should be applicable to SGR outbursts where rotational complications are generally absent. The next challenge is to understand the electrodynamics of black holes in much more detail. Of course this is a general relativistic calculation which must be carried out on a Kerr background. However, this aspect of the problem has been understood, at least in principle, as recent numerical simulations have demonstrated. It is the behavior of the gas and the magnetic field in and above the accretion disk that is still problematic.

However, it will still probably be too hard to compute forward from the region around an event horizon to the observable region in a relativistic jet, for example. It would therefore be very useful to have some more direct connection. Two possibilities suggest themselves. The first concerns the current flow. In the limiting case that the black hole and perhaps the inner disk drive a current along both jets, the sign of the radial current

is minus the the sign of $\Omega \cdot \vec{B}_{\text {disk }}$. This can be measured by seeking Faraday rotation gradients across the jet. (The line of sight field associated with the jet current will change sign across the jet.) If the gradient is maintained along a jet then this will imply 
a continuous current. If the gradient can be measured in both jets and it changes sign, then the component of $\vec{B}$ along $\vec{\Omega}$ at the disk must have the same sign on either side of the disk. In other words the jet field passes through the disk and the overall current completes with a radial disk current, Conversely, if the rotation measure gradient does not change sign then net radial field in the disk must continually be leaving through its top and the bottom surfaces and the current flow is dipolar (odd symmetry) and does not complete through the disk. The former pattern is what one expects with a pulsar where there is no disk. If one associates a current with dissipation - i.e. particle acceleration - then this may have already been seen in sources like the Crab Nebula where "disk" and "jet" morphological features can clearly be seen in the X-ray images. If the latter pattern is observed, then this is telling us something quite important about the behavior of accretion disks. Suppose that there is a net radial field associated with gas that is incorporated into the disk at its outer radius and this persists for several orbits. It could be associated with a single molecular cloud for example. Now, suppose that the disk is conservative in the sense that all of the radial flux remains trapped between the lower and upper surfaces as the gas spirals inward with ever shorter inflow timescales. It is easy to demonstrate that even though the stress associated with the horizontal field component will quickly dominate the other stresses and field lines must leave both disk surfaces creating a dipolar current pattern. There is a further implication of either measurement. The presence of a continuous current along both jets implies that the travel time along the jet is shorter than the characteristic mass (and magnetic flux) supply timescale at the outer radius. It is not hard to satisfy this condition if the disk extends for a million or so gravitational radii to the radius where the circular velocity is comparable with the central stellar velocity dispersion in the host galaxy. (Note that the flux supplied with a non-relativistically expanding cavity like a radio lobe or a supernova remnant must change sign over the lifetime of the source or escape through the cavity walls or, most plausibly, reconnect within a dissipative jet.)

The second possible connection is much less direct and more phenomenological. There is a view of AGN that their observable properties, after removing the effects of observer inclination which seriously affect beaming and obscuration, are essentially determined by the mass, which only provides a scale to the power, size, timescale, etc, the current mass supply rate (in units of the Eddington rate which is $\propto M$ ) and the spin angular frequency measure in units of its maximal allowed value $\propto m^{-1}$. Other factors that could in principle be important are the central stellar density, the morphological type of the host galaxy and so on. This view, which I have called the "Central Dogma"3, is testable observationally using large, homogeneous samples of AGN. It might be though that this is obviously wrong because powerful radio sources are associated with elliptical not spiral galaxies and preferentially with the most massive examples. However, if this is just a reflection of the nature of black holes in the nuclei of different types of galaxy which is, in turn, a reflection of the accretion history, then the Central Dogma can still be satisfied. Put differently, it says that intrinsic properties of AGN are determined by the current state of the black hole - accretion disk machine. Confirming or refuting this

\footnotetext{
3 After a similar successful conjecture in molecular biology
} 
conjecture would be a major advance in our understanding of AGN.

In this talk I have provided a broad though shallow overview of high energy astrophysics. and where it may be going. Given the current observational and computational opportunities at our disposal now, it ought to be possible to make great progress on understanding unifying and fundamental principles over the coming decade to match the progress that has been made in cosmology over the past decade.

\section{ACKNOWLEDGMENTS}

I thank Dr Kodama for the invitation to this symposium and Dr. Ioka for his help and patience with this manuscript. Several participants at this workshop along with my colleagues at KIPAC are thanked for their contributions to the ideas discussed above. Support by the U.S Department of Energy under contract number DE-AC02-76SF00515 is also gratefully acknowledged.

\section{REFERENCES}

1. S. Ritz, P. Michelson and C. Meegan The First GLAST Symposium AIP Conf. Proc. 921 (2007)

2. B. M. Gaensler and P. O. Slane. ARAA 4417 (2006)

3. P. Meszaros ARAA 40137 (2002)

4. T. A. Rector and D. S. De Young Extragalactic Jets: Theory and Observation from Radio to Gamma Ray A. S. P. Conference Series 386 (2008)

5. S. P. Reynolds, $A R A A, 46$ (in press) (2008).

6. S. E. Woosley and J. S. Bloom ARAA 44507 (2006) 